\title{
A MAN-MACHINE SYSTEM FOR SPATIAL INTERACTION ANALYSIS
}

\author{
Chien-Yuan Lin, Associate Professor \\ Chien-Hua Hwang, Research Assistant \\ Institute of Building and Planning \\ National Taiwan University, Taipei, Taiwan
}

\section{ABSTRACT}

Spatial interaction analysis is a task often required in geograph-related planning activities, such as transportation planning, urban planning and business marketing planning. Spatial interaction data are usually displayed in a matrix format. Transportation origin-destination (O-D) table and inter-regional population migration table are typical examples. To demonstrate the spatial characteristics of interaction data, maps have been the main presentation forms that are often used by planners. In order to support planners in the analysis process, a microcomputer-based program, INTERMAP, is developed to form a man-machine system for spatial interaction analysis. In this paper, the development and system features of INTERMAP are described.

Keywords: Spatial Interaction Analysis, OriginDestination Map, Computer Graphics

\section{INTRODUCTION}

Analysis of spatial interaction data is an important task for geograph-related planning activities. For example, urban and transportation planners need to identify spatial interaction patterns among traffic analysis zones (TAZ) and social planners need to explore inter-regional migration patterns. Usually, spatial interaction data are presented in a matrix format, transportation origin-destination (O-D) table in transportation and inter-regional population migration matrix are typical examples. Table 1 shows a $4 \times 4$ population migration data of 1988 among regions in Taiwan. Since the problem complexity will be exponentially expanded when the number of zones increases, methods to help planners analyze interaction data are urgently needed. How to develop a man-machine system for planners to conduct spatial interaction analysis efficiently and effectively is a challenging task in computer applications.

From our literature review, we found that a couple of application programs have been specially developed for O-D map preparation, however.
Table 1 Matrix of Inter-Regional Population Migration

\begin{tabular}{|c|c|c|c|c|}
\hline & 1 & 2 & 3 & 4 \\
\hline 1 & 355231.00 & 103327.00 & 127536.00 & 19465.00 \\
\hline 2 & 166801.00 & 93899.00 & 36429.00 & 4961.00 \\
\hline 3 & 226443.00 & 17342.00 & 171208.00 & 2842.00 \\
\hline 4 & 5715.00 & 2082.00 & 2571.00 & 7892.00 \\
\hline
\end{tabular}

neither of them has provided both interactive data editing and analysis capabilities while compatibility with other application systems are kept. FLOWMAP is the first system developed in 1979 at the University of Washington, Seattle, U.S.A. [1]. The system is programmed in FORTRAN IV with PLOT-10 graphic template on Tektronix 4014 graphic terminal connecting to CDC mainframe computer. Hardcopy can be obtained by a Tektronix copier. Due to its hardware dependence, distribution of FLOWMAP has been very limited. In fact, FLOWMAP was phased out when the University of Washington shut down its Cyber system in 1989 .

SPACEMAT (Spatial Matrix Display and Manipulation) is another system for the same purpose developed on PC/AT by Craford and Simberg in Australia in 1983. [3]. In SPACEMAT, the matrix display and analysis was performed by representing each cell in the data matrix as a block of color the color corresponding to a data class interval chosen by the user and easily (interactively) changed by him. The horizontal axis represents, in order, the destination zones of work trips while the vertical axis represents the origin zones, and the color coding can represent total trips. Immediately, the whole of the (usually) large origin-destination (O-D) matrix can be inspected for patterns, or the user may interactively regroup the data into different interval classes and rapidly redisplay in the resultant matrix. However, the generated $O-D$ map is a schematic matrix instead of a map with geographic feature. 
Recognizing the need of a man-machine system for spatial interaction analysis, we began to develop an interactive computer graphic system at the Graduate Institute of Building and Planning, National Taiwan University since 1988 [2]. The result system is named INTERMAP and the latest version is 2.1 .

\section{SYSTEM DESIGN PRINCIPLES}

Any piece of data item may contain three different attributes, namely, "time", "space" and "thematic". When most data are defined with focus on "time" and "thematic" attributes, spatial interaction data are particularly important of its spatial attribute. To deal with spatial data analysis, traditional methods have focused on multivariate statistic analysis and input-output matrix manipulation. Although O-D maps have been recognized excellent presentation media for spatial interaction data, analysis through graphic approach has been very limited due to the lack of effective and efficient tool. The main objective of INTERMAP is to pave a way for planners in spatial interaction analysis via graphic approach that takes advantage of state-of-art computer graphic techniques.

To obtain this objective, several design principles are predefined for the development of INTERMAP. Each principle is briefly described as follows:

1. Interactive response. To be an effective tool for data analysis, capability for fast interactive response is absolutely required.

2. Graphical what-if analysis. What-if analysis is a key element for various planning activities. While multivariate statistic methods and inputoutput matrix manipulations have focused on data reduction and relationship identification, graphical what-if analysis will give an excellent chance to explore spatial interaction patterns and to identify key interaction elements in a huge numeric matrix.

3. Data manipulation and editing. In most data analysis systems, input data are often required to be prepared and edited outside the system, which forces users to switch between different application programs in data preparation and manipulation. To keep data editing and data analysis in the same system is one of the features defined for system development.

4. Desktop publishing capabilities. One of the jobs that planners need to do is to present analysis results in a quality report to decision makers. To generate quality O-D map from spatial analysis, desktop publishing capability has to be provided for graphic and text editing.

5. System flexibility. INTERMAP is intended to be one of application programs that will be used by planners. Recognizing the fact that planners may need to import data from other application programs and to export output results to other programs for further analysis or editing, the system will have flexibility in input/output interface with other application programs. Besides, since the map readability will be deteriorated when more O-D pairs are involved, different graphical analysis methods need be provided.

6. User friendly system. Obviously, most planners only have very little of computer knowledge. To be an effective analytical tool for planners to apply, user-friendliness is a must for the manmachine system so that man-machine interaction can be easily processed.

\section{SYSTEM OPERATIONAL REQUIREMENTS}

The system operational requirements of INTERMAP are described in terms of hardware configuration, software and input data preparation respectively as follows:

\section{A. Hardware Configuration}

PC/AT or compatible computer with at least 640K memory, Hercules monitor, VGA or EGA monitors all are acceptable. A mouse is preferred for INTERMAP operation. If hardcopy is desired during operation process, a HP Laserjet II compatible laser printer is a must. Digitizer and pen plotter are optional.

B. Software Requirement

INTERMAP version 2.1 is an independent application program that can be operated without any other supporting programs. However, if an user desires to digitize a map or plot out a map under the same system, then AutoCAD is required. The capability of DXF files processing has provided INTERMAP an excellent independent interface for graphic processing.

\section{Data Input Requirement}

A file of origin-destination (O-D) matrix is required to provide the basis of interaction analysis. O-D data can be input directly through data editing function in INTERMAP or worksheet prepared by LOTUS 1-2-3. If geographic map is to be displayed, then a digitized boundary file is also required. Digitizing can be done by the digitizing program provided by INTERMAP or computer aided design system AUTOCAD. Coordinates for zonal centroids can be obtained during operation session. 


\section{DESIGN OF MAN-MACHINE INTERFACE}

It is widely recognized that interface design is the key determinant for a man-machine system. In order to facilitate planners in the man-machine interactive analysis process, many interfaces to enhance planner's analytical capability are provided. The most important four of them are briefly described in that which follows:

1. Interface for data extraction. In the analysis of matrix data, the most important task for an analyst is to identify where are the data of his/her interest. For instance, a transportation planner would be interested in identifying O-D pairs with major traffic flow. INTERMAP has provided an data extraction interface that allows user specify the upper bound and lower bound of the data for further graphic analysis. By the same interface, user can easily find out what proportion his/her extracted data occupies in the total flow volume. Figure 1 shows the data extraction interface.

2. Interface for data classification. In order to simplify the data for graphic display, Figure 2 show an interface that user can use to perform data classification easily. Through which, user has flexibility to specify how many intervals to be segmented and which interval method to be used, such as equal-distribution, equal-distance or random-specification method. User can identify the spatial characteristics of data since INTERMAP will display the classified matrix data by coloring or shading scheme. Figure 3 is an example of classified data display.

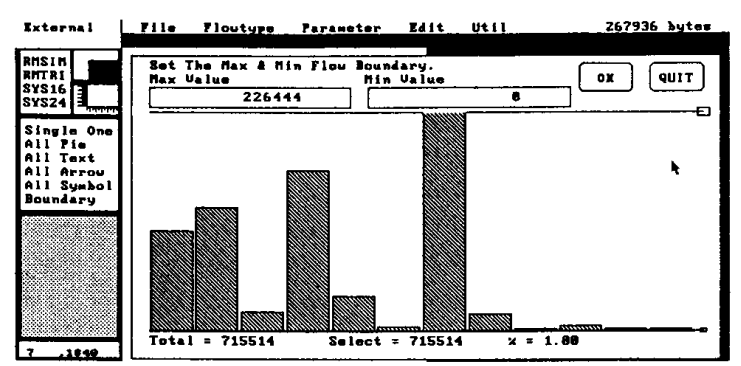

Figure 1 Interface for Data Extraction

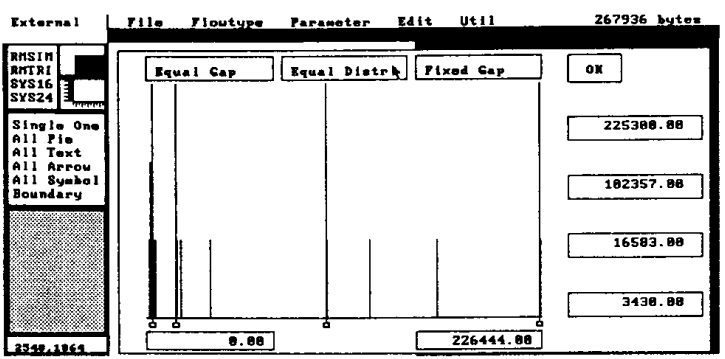

Figure 2 Interface for Data Classification
3. Interface for zone selection. Figure 4 shows an interface that user may select zones for analysis. So that the graphic analysis can be easily performed for one-to-many, many-to-one, or many-to-many analysis.

4. Graphic editing interface. An interface with desktop publishing capability for graphic editing shown as Figure 5 is provided in INTERMAP. Based on the auto-generated map in the dataanalysis session, user may further editing the graphic in order to obtain quality output graph. The graphic editing elements include options for Line Type, Shading, Text, Arrow Type, Zooming, Pan and Move.

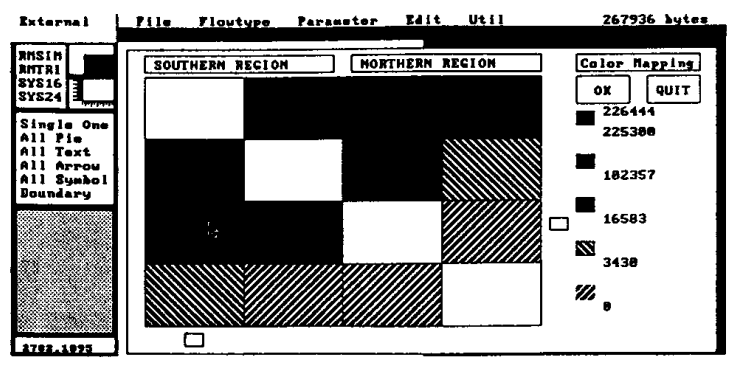

Figure 3 Result Matrix of Data Classification

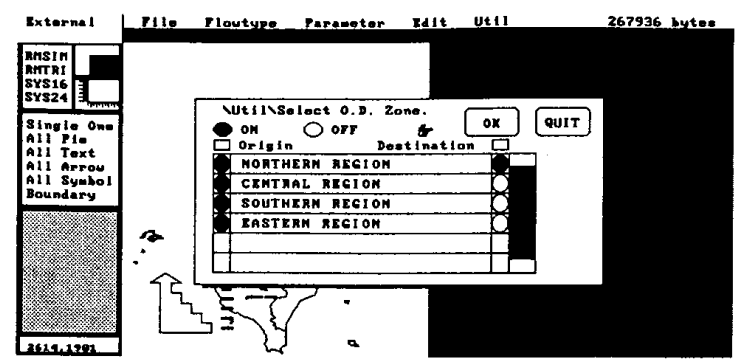

Figure 4 Interface for Zone Selection

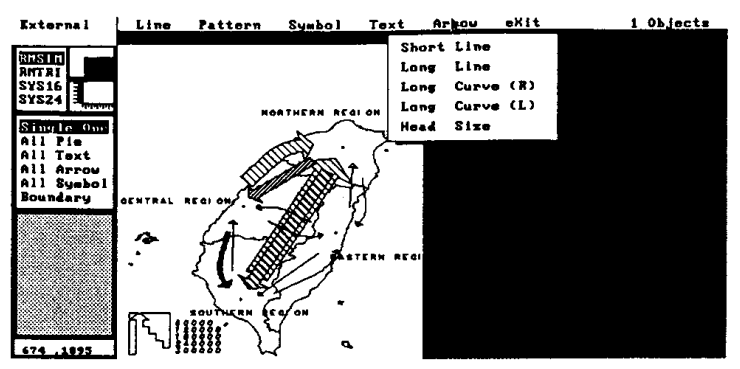

Figure 5 Interface for Graphic Editing 


\section{SYSTEM CAPABILITIES IN INTERMAP}

To support planners in spatial interaction analysis, INTERMAP is to provide an interactive analysis environment in addition to its computer aided drafting function. In other words, INTERMAP not only helps users in O-D map preparation, it also help planners "think" problem in their analysis process by providing a variety of mapping styles and map editing functions.

Basically, arrow and pie are the two graphic primitives used in INTERMAP to demonstrate the spatial interaction characteristics. In general, six different types of O-D maps can be prepared by INTERMAP.

1. Inter-zonal flow values can be represented by arrows with different width, width of each arrow is proportional to its flow volume. Figure 6 shows an example of this type.

2. Intra-zonal flow (origin and destination are within the same zone) can be represented by pie, radius of pie is proportional to its volume.

3. Net-flow for each O-D pair (difference between in-flow and out-flow) can be represented by arrow with varying width, the arrow head points to the end with more in-flow values. Figure 7 is a net-flow map for Figure 6.

4. Origin piegraph, area of pie is proportional to the total flow volume originated from each zone, where shaded area represents the proportion of intra-zonal flow. Figure 8 is an example.

5. Destination piegraph, similar to origin piegraph but representing flows destinated at each zone, where intrazonal flows are still shaded proportionally.

6. Arrows and pies are displayed simultaneously on the same graph such as example in Figure 6.

In order to explore interaction relationships for different number of O-D pairs, INTERMAP allows users to select zones of interest for analysis, so that interaction zones in a map may be displayed in many-to-many, many-to-one, or oneto-many styles. If the user has no specific selection of zones for analysis, all zones are displayed in a many-to-many format. In many-tomany O-D maps, more than one zone are selected for origin and destination zones separately, while there are only one origin or one destination selected for one-to-many or many-to-one analysis. The flexibility of zone selection has provided users a powerful tool in identifying spatial relationships for interesting O-D pairs easily. Another useful function provided in INTERMAP is that it allows user select O-D pairs with flow values within a certain intervals. For instances, an user may only choose O-D pairs that have flow values greater than a certain level.
Inter-zonal Migration $\ln 1988$

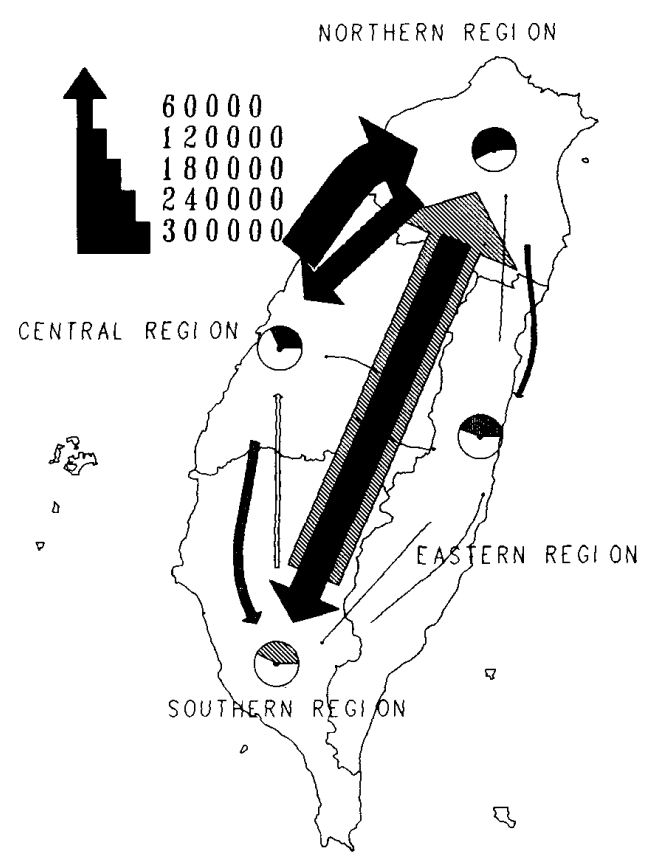

Figure 6 Map of Many-to-Many Interzonal Interaction

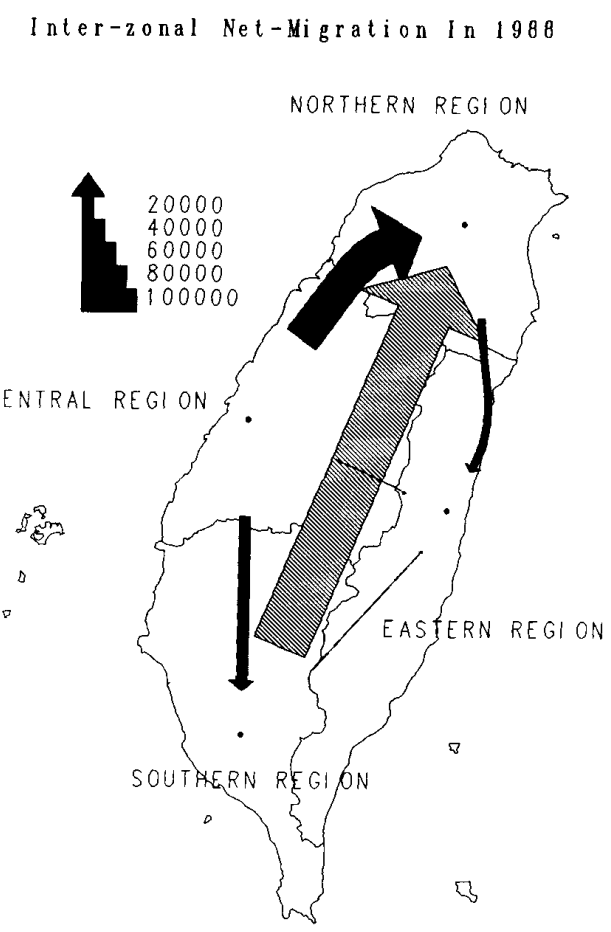

Figure 7 Map of Netflow Interaction 
The Traffic Flows of HSINCHU in 1990

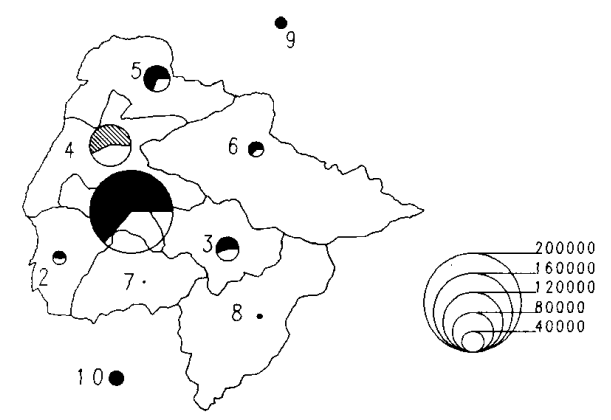

Figure 8 Map of Piegeaph

\section{SYSTEM STRUCTURE AND OPERATION STYLE}

Essentially, INTERMAP is composed of four functional modules for matrix preparation, map digitizing, map editing and graphic output separately. Functions for each module are briefly described as follows:

\section{A. Matrix Preparation Module}

An O-D matrix is the basis for INTERMAP operation. The O-D matrix may represent traffic flows among traffic analysis zones, or population migration among each administration area, or any other type of spatial interaction data that can be presented in a matrix format. Since the style of spreadsheet operation is very appropriate for matrix manipulation, a simple spreadsheet-like program is designed to serve as the front-end interface for matrix preparation. In addition, an utility program is provided to read LOTUS worksheet directly for INTERMAP operation. The maximum matrix size that can be handled is 50 by 50 .

\section{B. Digitizing Module}

Map digitizing is a required process for producing electronic map. Coordinate data can be digitized either through application programs such as AutoCAD, or other independent digitizing program. If map is digitized by AutoCAD, the drawing file has to be saved in Data Exchange File (DXF) format for use in INTERMAP. Two types of coordinate data are required in INTERMAP operation. One is boundary data, the other includes coordinate data for zone centroids and labels. Only the former type need to be prepared before INTERMAP execution, coordinates for the second type can be picked from the screen directly. However, it should be noticed that coordinate data may be neglected if no map boundary is to be displayed. For instance, INTERMAP is used to analyze interaction data among some hypothetical nodes without physical locations in the space.

\section{Map Editing Module}

This is the core module in this system, where user may produce/edit an O-D map, prepare histogram, retrieve/save files and produce hardcopy or data interchange files for AutoCAD. When INTERMAP is invoked, a many-to-many O-D map will be generated for all interaction flows on the matrix. Based on the automatically generated draft, user may improve the map by changing arrow type, width, outbound/inbound radius, pie radius, shading pattern, centroid symbol, moving graphic objects and labels. Edited graphs can be printed out or saved together with O-D matrix as a job for further improvement.

\section{Graph Output Module}

When a satisfied O-D map is designed during the map editing process, hardcopy can be obtained through laser printer directly. A satisfied $O-D$ map not only has clear graphic layout, but also provides information that the analyst wants to express through map. Besides, map can be printed out as a data interchange file (DXF) for AutoCAD so that it can be further edited in AutoCAD, especially when users prefer to have color hardcopy or inserting additional graphic units. Output in DXF format also relieves the system from preparing drivers for different output devices.

In order to provide users a friendly environment in spatial interaction analysis process so that features for a given O-D matrix can be easily identified through graphic manipulation, Macintosh-like interface style is adopted in INTERMAP. Based on pull down menu driven style, with a mouse and minimum typing effort, the user is expected to proceed easily through each stages even if one does not have computer experience.

\section{CONCLUSION}

A map is worth more than thousands of words, this is especially true for planners, such as urban and transportation planners, who have to deal with spatial data analysis. In this paper, we have described a man-machine system, INTERMAP, that could be used to help planners generate and analyze spatial interaction data more efficiently and effectively. Version 2.1 is the latest copy of INTERMAP's development. 
There are so many potential application areas that INTERMAP can be used to facilitate the process of spatial interaction analysis. Some are briefly introduced as follows:

1. Transportation planning : Passenger and freight transportation patterns are often surveyed at different geographic area and displayed in a matrix format. By use of INTERMAP, these O-D tables can be easily displayed by O-D map so that it can be easily comprehended by transportation planners and decision makers.

2. Public facility planning: Public facilities are major traffic attraction locations in a city. To analyze the spatial distribution pattern of their users and their impact on other facilities, INTERMAP can be used to help planner identify of spatial distribution pattern.

3. Population study: Population study is a key sector in various social and economic planning activities. O-D map will be an effective tool to explore the population migration pattern among regions so that social and economic development strategies can be formulated.

4. Economic analysis: To identify the inter-regional economic interaction pattern, economic inputoutput table are prepared by region and economic sectors respectively. O-D map can be used to display their spatial interaction pattern more clearly. In fact, analysis of international trade patterns would be an ideal example.

5. Market analysis: PCFLOW's use is not confined for spatial data only. Figure 5 shows consumption patterns for two different brands of product in different regions. The use of hypothetical nodes makes INTERMAP a powerful market analysis tool.

INTERMAP is mainly consisted of four functional modules, namely, matrix preparation, digitizing, graphic editing and graphic output modules. The major contribution of INTERMAP is the provision of an user-friendly environment for planners to design high quality $O-D$ maps and analyze spatial interaction patterns through graphical what-if analysis so that complex interrelationships can be easily identified. More specifically, the man-machine system has integrated the numeric matrix analysis with graphic analysis capability. Besides, INTERMAP is also featured in its integration of Computer Aided Design and Desktop Publishing techniques for spatial data analysis. Future developments of INTERMAP will be focused on the extension of system capabilities in the above-mentioned potential application areas.

\section{REFERENCE}

[1] Evatt, B.S. Jr.; Schneider, J.B. and Greeberg, H., "Mapping Origin-Destination Patterns in Space and Time by Using Interactive Computer Graphics", Iransportation Research Becord, No.866, pp.25-35, 1982

[2] Lin, Chien-yuan, Hwang, Chien-Hua, and Wang, Chih-hui, "An Interactive Mapping System for Spatial Interaction Analysis", Proceedings of the ISMM International Symposium, COMPUTER APPLICATIONS IN DESIGN, SIMULATION AND ANALYSIS, MIMI'90 held in New Orleans, U.S.A., March 5-7, 1990, pp.120-123, ACTA Press

[3] Newton, P. W. and Crawford, J. R. "Microcomputer-Based Geographic Information and Mapping Analysis", in Newton, P.W. (eds) Desktop Planning pp.31-43, Hargreen Publishing Company, Melbourne, 1988

[4] Yeh, A. Gar-on, "Microcomputers in Urban Planning: Applications, Constraints, and Impacts", Environment and Planning B: Planning and Design, Vol.15, No.3, pp.241-254, 1988 